PROCEEDINGS OF THE

AMERICAN MATHEMATICAL SOCIETY

Volume 128, Number 10, Pages 2989-2997

S 0002-9939(00)05530-1

Article electronically published on April 28, 2000

\title{
OSCILLATION CRITERIA FOR DELAY EQUATIONS
}

\author{
M. KON, Y. G. SFICAS, AND I. P. STAVROULAKIS
}

(Communicated by Hal L. Smith)

Dedicated to Professor V. A. Staikos on the occasion of his 60th birthday

AbStRact. This paper is concerned with the oscillatory behavior of first-order delay differential equations of the form

$$
x^{\prime}(t)+p(t) x(\tau(t))=0, \quad t \geq t_{0},
$$

where $p, \tau \in C\left(\left[t_{0}, \infty\right), \mathbb{R}^{+}\right), \mathbb{R}^{+}=[0, \infty), \tau(t)$ is non-decreasing, $\tau(t)<t$ for $t \geq t_{0}$ and $\lim _{t \rightarrow \infty} \tau(t)=\infty$. Let the numbers $k$ and $L$ be defined by

$$
k=\liminf _{t \rightarrow \infty} \int_{\tau(t)}^{t} p(s) d s \text { and } L=\limsup _{t \rightarrow \infty} \int_{\tau(t)}^{t} p(s) d s .
$$

It is proved here that when $L<1$ and $0<k \leq \frac{1}{e}$ all solutions of Eq. (1) oscillate in several cases in which the condition

$$
L>2 k+\frac{2}{\lambda_{1}}-1
$$

holds, where $\lambda_{1}$ is the smaller root of the equation $\lambda=e^{k \lambda}$.

\section{INTRODUCTION}

The problem of establishing sufficient conditions for the oscillation of all solutions of the differential equation

$$
x^{\prime}(t)+p(t) x(\tau(t))=0, \quad t \geq t_{0},
$$

where the functions $p, \tau \in C\left(\left[t_{0}, \infty\right), \mathbb{R}^{+}\right)$(here $\left.\mathbb{R}^{+}=[0, \infty)\right), \tau(t)$ is nondecreasing, $\tau(t)<t$ for $t \geq t_{0}$ and $\lim _{t \rightarrow \infty} \tau(t)=\infty$, has been the subject of many investigations. See, for example, [1]-[26] and the references cited therein.

By a solution of Eq. (1) we understand a continuously differentiable function defined on $\left[\tau\left(T_{0}\right), \infty\right)$ for some $T_{0} \geq t_{0}$ and such that (1) is satisfied for $t \geq T_{0}$. Such a solution is called oscillatory if it has arbitrarily large zeros, and otherwise it is called nonoscillatory.

The first systematic study for the oscillation of all solutions of Eq. (1) was made by Myshkis. In 1950 [23] he proved that every solution of Eq. (1) oscillates if

$$
\limsup _{t \rightarrow \infty}[t-\tau(t)]<\infty, \quad \liminf _{t \rightarrow \infty}[t-\tau(t)] \cdot \liminf _{t \rightarrow \infty} p(t)>\frac{1}{e} .
$$

Received by the editors December 4, 1998.

1991 Mathematics Subject Classification. Primary 34K15; Secondary 34C10.

Key words and phrases. Oscillation, delay differential equations. 
In 1972, Ladas, Lakshmikantham and Papadakis [18 proved that the same conclusion holds if

$$
\limsup _{t \rightarrow \infty} \int_{\tau(t)}^{t} p(s) d s>1
$$

In 1979 Ladas [17] and in 1982 Koplatadze and Chanturija [13] improved $\left(C_{1}\right)$ to

$$
\liminf _{t \rightarrow \infty} \int_{\tau(t)}^{t} p(s) d s>\frac{1}{e}
$$

Concerning the constant $\frac{1}{e}$ in $\left(C_{3}\right)$, it is to be pointed out that if the inequality

$$
\int_{\tau(t)}^{t} p(s) d s \leq \frac{1}{e}
$$

holds eventually, then, according to a result in [13], (1) has a non-oscillatory solution.

In 1982 Ladas, Sficas and Stavroulakis [19] and in 1984 Fukagai and Kusano [10] established oscillation criteria (of the type of conditions $\left(C_{2}\right)$ and $\left(C_{3}\right)$ ) for Eq. (1) with oscillating coefficient $p(t)$.

It is obvious that there is a gap between the conditions $\left(C_{2}\right)$ and $\left(C_{3}\right)$ when the limit

$$
\lim _{t \rightarrow \infty} \int_{\tau(t)}^{t} p(s) d s
$$

does not exist. How to fill this gap is an interesting problem which has been recently investigated by several authors.

In 1988, Erbe and Zhang [9] developed new oscillation criteria by employing the upper bound of the ratio $x(\tau(t)) / x(t)$ for possible nonoscillatory solutions $x(t)$ of Eq. (1). Their result, when formulated in terms of the numbers $k$ and $L$ defined by

$$
k=\liminf _{t \rightarrow \infty} \int_{\tau(t)}^{t} p(s) d s \text { and } L=\limsup _{t \rightarrow \infty} \int_{\tau(t)}^{t} p(s) d s,
$$

says that all the solutions of Eq. (1) are oscillatory, if $0<k \leq \frac{1}{e}$ and

$$
L>1-\frac{k^{2}}{4}
$$

Since then several authors tried to obtain better results by improving the upper bound for $x(\tau(t)) / x(t)$. In 1991 Jian Chao 2] derived the condition

$$
L>1-\frac{k^{2}}{2(1-k)},
$$

while in $1992 \mathrm{Yu}$ and Wang [25] and Yu, Wang, Zhang and Qian [26] obtained the condition

$$
L>1-\frac{1-k-\sqrt{1-2 k-k^{2}}}{2} .
$$

In 1990 Elbert and Stavroulakis [7] and in 1991 Kwong [16], using different techniques, improved $\left(C_{4}\right)$, in the case where $0<k \leq \frac{1}{e}$, to the conditions

$$
L>1-\left(1-\frac{1}{\sqrt{\lambda_{1}}}\right)^{2}
$$


and

$$
L>\frac{\ln \lambda_{1}+1}{\lambda_{1}},
$$

respectively, where $\lambda_{1}$ is the smaller root of the equation

$$
\lambda=e^{k \lambda} .
$$

In 1994 Koplatadze and Kvinikadze [14] improved $\left(C_{6}\right)$, while in 1996 Philos and Sficas 24] and Jaroš and Stavroulakis [11] derived the conditions

$$
L>1-\frac{k^{2}}{2(1-k)}-\frac{k^{2}}{2} \lambda_{1}
$$

and

$$
L>\frac{\ln \lambda_{1}+1}{\lambda_{1}}-\frac{1-k-\sqrt{1-2 k-k^{2}}}{2}
$$

respectively, where $\lambda_{1}$ is the smaller root of Eq. (2).

Following this historical (and chronological) review we also mention that in the case where

$$
\int_{\tau(t)}^{t} p(s) d s \geq \frac{1}{e} \text { and } \lim _{t \rightarrow \infty} \int_{\tau(t)}^{t} p(s) d s=\frac{1}{e}
$$

this problem has been studied in 1993 by Elbert and Stavroulakis [8] and in 1995 by Kozakiewicz [15], Li [21, 22] and by Domshlak and Stavroulakis [5].

The purpose of this paper is to improve the methods previously used to show that in several cases the conditions $\left(C_{2}\right)$ and $\left(C_{4}\right)-\left(C_{10}\right)$ may be weakened to

$$
\left(C_{11}\right) \quad L>2 k+\frac{2}{\lambda_{1}}-1,
$$

where $\lambda_{1}$ is the smaller root of the equation $\lambda=e^{k \lambda}$.

It is to be noted that as $k \rightarrow 0$, then all conditions $\left(C_{4}\right)-\left(C_{10}\right)$ and also our condition $\left(C_{11}\right)$ reduce to the condition $\left(C_{2}\right)$. However the improvement is clear as $k \rightarrow \frac{1}{e}$. For illustrative purposes, we give the values of the lower bound on $L$ under these conditions when $k=\frac{1}{e}$ :

$\begin{array}{ll}\left(C_{2}\right): & 1.000000000 \\ \left(C_{4}\right): & 0.966166179 \\ \left(C_{5}\right): & 0.892951367 \\ \left(C_{6}\right): & 0.863457014 \\ \left(C_{7}\right): & 0.845181878 \\ \left(C_{8}\right): & 0.735758882 \\ \left(C_{9}\right): & 0.709011646 \\ \left(C_{10}\right): & 0.599215896 \\ \left(C_{11}\right): & 0.471517764\end{array}$

We see that our condition $\left(C_{11}\right)$ essentially improves all the known results in the literature.

\section{MAin RESUlts}

In what follows we will denote by $k$ and $L$ the lower and upper limits of the average $\int_{\tau(t)}^{t} p(s) d s$ as $t \rightarrow \infty$, respectively, i.e.

$$
k=\liminf _{t \rightarrow \infty} \int_{\tau(t)}^{t} p(s) d s
$$


and

$$
L=\limsup _{t \rightarrow \infty} \int_{\tau(t)}^{t} p(s) d s
$$

Set

$$
w(t)=\frac{x(\tau(t))}{x(t)}
$$

We begin with the preliminary analysis of asymptotic behavior of the function $w(t)$ for a possible nonoscillatory solution $x(t)$ of Eq. (1) in the case that $k \leq \frac{1}{e}$. For this purpose, assume that (1) has a solution $x(t)$ which is positive for all large $t$. Dividing first Eq. (1) by $x(t)$ and then integrating it from $\tau(t)$ to $t$ leads to the integral equality

$$
w(t)=\exp \int_{\tau(t)}^{t} p(s) w(s) d s
$$

which holds for all sufficiently large $t$.

For the next lemma see 11.

Lemma 1. Suppose that $k>0$ and Eq. (1) has an eventually positive solution $x(t)$. Then $k \leq 1 / e$ and

$$
\lambda_{1} \leq \liminf _{t \rightarrow \infty} w(t) \leq \lambda_{2}
$$

where $\lambda_{1}$ is the smaller and $\lambda_{2}$ the greater root of the equation $\lambda=e^{k \lambda}$.

Proof. Let $\alpha=\liminf _{t \rightarrow \infty} w(t)$. From (3) we have for sufficiently large $t$ that

$$
\alpha \geq \exp k \alpha
$$

which is impossible if $k>1 / e$, since a simple calculus argument shows that in this case $\lambda<e^{k \lambda}$ for all $\lambda$. This implies that (1) has no eventually positive solution if $k>1 / e$. On the other hand, if $0<k \leq 1 / e$, then $\lambda=e^{k \lambda}$ has $\operatorname{roots} \lambda_{1} \leq \lambda_{2}$ (with equality $\lambda_{1}=\lambda_{2}=e$ if and only if $k=1 / e$ ), and $\alpha \geq e^{k \alpha}$ if and only if $\lambda_{1} \leq \alpha \leq \lambda_{2}$

Lemma 2. Let $0<k \leq \frac{1}{e}$ and $x(t)$ be an eventually positive solution of Eq. (1). Assume that there exists $\theta>0$ such that

$$
\int_{\tau(u)}^{\tau(t)} p(s) d s \geq \theta \int_{u}^{t} p(s) d s \text { for all } \tau(t) \leq u \leq t .
$$

Then

$$
\limsup _{t \rightarrow \infty} w(t) \leq \frac{2}{1-k-\sqrt{(1-k)^{2}-4 A}},
$$

where $A$ is given by

$$
A=\frac{e^{\lambda_{1} \theta k}-\lambda_{1} \theta k-1}{\left(\lambda_{1} \theta\right)^{2}} .
$$

Proof. Let $\delta: 0<\delta<k$ be any number arbitrarily close to $k$ and $T>t_{0}$ large enough so that $\tau(t)>t_{0}$ and $\int_{\tau(t)}^{t} p(s) d s>\delta$ for every $t \geq T$. Let $t \geq T$ and $T_{1} \equiv T_{1}(t)>t: \tau\left(T_{1}\right)=t$. Since $\int_{t}^{T_{1}} p(s) d s>\delta$ there exists $T_{1}>t_{1} \equiv t_{1}(t)>t$ 
such that

$$
\int_{t}^{t_{1}} p(s) d s=\delta
$$

Integrating (1) from $t$ to $t_{1}$, we obtain

$$
x(t)=x\left(t_{1}\right)+\int_{t}^{t_{1}} p(s) x(\tau(s)) d s,
$$

while integrating from $\tau(s)$ to $t$ for $s<t_{1}$, we have

$$
x(\tau(s))=x(t)+\int_{\tau(s)}^{t} p(u) x(\tau(u)) d u .
$$

Combining the last two equalities, we obtain

$$
x(t)=x\left(t_{1}\right)+\int_{t}^{t_{1}} p(s)\left[x(t)+\int_{\tau(s)}^{t} p(u) x(\tau(u)) d u\right] d s .
$$

Let $0<\lambda<\lambda_{1}$. Then the function

$$
\varphi(t)=x(t) e^{\lambda \int_{t_{0}}^{t} p(s) d s}, \quad t \geq a,
$$

is decreasing for appropriate $a \geq t_{0}$ since $x(t)$ is also decreasing. Indeed, by Lemma 1 ,

$$
\frac{x(\tau(t))}{x(t)}>\lambda
$$

for all sufficiently large $t$, and consequently

$$
0=x^{\prime}(t)+p(t) x(\tau(t)) \geq x^{\prime}(t)+\lambda p(t) x(t)
$$

which implies $\varphi^{\prime}(t) \leq 0$ for sufficiently large $t$.

Substituting into (7), we derive for sufficiently large $t$

$$
\begin{aligned}
& x(t) \geq x\left(t_{1}\right)+\delta x(t)+\varphi(\tau(t)) \int_{t}^{t_{1}} p(s)\left(\int_{\tau(s)}^{t} p(u) e^{-\lambda \int_{t_{0}}^{\tau(u)} p(\xi) d \xi} d u\right) d s \\
= & x\left(t_{1}\right)+\delta x(t)+\varphi(\tau(t)) e^{-\lambda \int_{t_{0}}^{\tau(t)} p(s) d s} \int_{t}^{t_{1}} p(s)\left(\int_{\tau(s)}^{t} p(u) e^{\lambda \int_{\tau(u)}^{\tau(t)} p(\xi) d \xi} d u\right) d s
\end{aligned}
$$

and therefore

(8)

$$
x(t) \geq x\left(t_{1}\right)+\delta x(t)+x(\tau(t)) \int_{t}^{t_{1}} p(s)\left(\int_{\tau(s)}^{t} p(u) e^{\lambda \int_{\tau(u)}^{\tau(t)} p(\xi) d \xi} d u\right) d s .
$$

In view of (4), we obtain

$$
\begin{aligned}
\int_{\tau(s)}^{t} p(u) e^{\lambda \int_{\tau(u)}^{\tau(t)} p(\xi) d \xi} d u & \geq \int_{\tau(s)}^{t} p(u) e^{\lambda \theta \int_{u}^{t} p(\xi) d \xi} d u \\
& =\frac{1}{\lambda \theta}\left[e^{\lambda \theta \int_{\tau(s)}^{t} p(\xi) d \xi}-1\right]
\end{aligned}
$$


Thus

$$
\begin{aligned}
& \int_{t}^{t_{1}} p(s)\left(\int_{\tau(s)}^{t} p(u) e^{\lambda \int_{\tau(u)}^{\tau(t)} p(\xi) d \xi} d u\right) d s \geq-\frac{\delta}{\lambda \theta}+\frac{1}{\lambda \theta} \int_{t}^{t_{1}} p(s) e^{\lambda \theta \int_{\tau(s)}^{t} p(\xi) d \xi} d s \\
&=-\frac{\delta}{\lambda \theta}+\frac{1}{\lambda \theta} \int_{t}^{t_{1}} p(s) e^{\lambda \theta \int_{\tau(s)}^{s} p(\xi) d \xi-\lambda \theta \int_{t}^{s} p(\xi) d \xi} d s \\
& \geq-\frac{\delta}{\lambda \theta}+\frac{1}{\lambda \theta} e^{\lambda \theta \delta} \int_{t}^{t_{1}} p(s) e^{-\lambda \theta \int_{t}^{s} p(\xi) d \xi} d s \\
&=-\frac{\delta}{\lambda \theta}+\frac{e^{\lambda \theta \delta}}{(\lambda \theta)^{2}}\left[1-e^{-\lambda \theta \int_{t}^{t_{1}} p(\xi) d \xi}\right] \\
&=-\frac{\delta}{\lambda \theta}+\frac{e^{\lambda \theta \delta}}{(\lambda \theta)^{2}}\left[1-e^{-\lambda \theta \delta}\right]=-\frac{\delta}{\lambda \theta}+\frac{1}{(\lambda \theta)^{2}}\left(e^{\lambda \theta \delta}-1\right),
\end{aligned}
$$

and (8) yields

$$
x(t) \geq x\left(t_{1}\right)+\delta x(t)+A^{*} x(\tau(t))
$$

where

$$
A^{*}=\frac{e^{\lambda \theta \delta}-\lambda \theta \delta-1}{(\lambda \theta)^{2}}
$$

From (9), we have

$$
x(t) \geq d_{1} x(\tau(t)),
$$

where we have set

Observe that

$$
d_{1}=\frac{A^{*}}{1-\delta}
$$

$$
x\left(t_{1}\right) \geq d_{1} x\left(\tau\left(t_{1}\right)\right) \geq d_{1} x(t)
$$

since $x(t)$ is decreasing, and therefore (9) yields

$$
x(t) \geq d_{2} x(\tau(t))
$$

where

$$
d_{2}=\frac{A^{*}}{1-d_{1}-\delta} .
$$

Following this iterative procedure (cf. [25], 26]), we obtain

$$
x(t) \geq d_{n+1} x(\tau(t)),
$$

where

$$
d_{n+1}=\frac{A^{*}}{1-d_{n}-\delta}, \quad n=1,2, \ldots
$$

It is easy to see that the sequence $\left\{d_{n}\right\}$ is strictly increasing and bounded.

Therefore $\lim _{n \rightarrow \infty} d_{n}=d$ exists and satisfies

$$
d^{2}-(1-\delta) d+A^{*}=0
$$

Since $\left\{d_{n}\right\}$ is strictly increasing, it follows that

$$
d=\frac{1-\delta-\sqrt{(1-\delta)^{2}-4 A^{*}}}{2}
$$

Thus, for all large $t$

$$
\frac{x(t)}{x(\tau(t))} \geq \frac{1-\delta-\sqrt{(1-\delta)^{2}-4 A^{*}}}{2}
$$


and since $0<\delta<k$ is arbitrarily close to $k$, by letting $\lambda \rightarrow \lambda_{1}$ the last inequality leads to (5). The proof is complete.

Remark 1. Assume that $\tau(t)$ is continuously differentiable and that there exists $\theta>0$ such that

$$
p(\tau(t)) \tau^{\prime}(t) \geq \theta p(t)
$$

eventually for all $t$. Then it is easy to see that (10) implies (4). Indeed, the function

$$
v(u)=\int_{\tau(u)}^{\tau(t)} p(s) d s-\theta \int_{u}^{t} p(s) d s, \quad \tau(t) \leq u \leq t,
$$

satisfies the conditions

$$
v(t)=0
$$

and

$$
v^{\prime}(u)=-p(\tau(u)) \tau^{\prime}(u)+\theta p(u) \leq 0 .
$$

If $p(t)>0$ eventually for all $t$ and

$$
\liminf _{t \rightarrow \infty} \frac{p(\tau(t)) \tau^{\prime}(t)}{p(t)}=\theta_{0}>0,
$$

then $\theta$ can be any number satisfying $0<\theta<\theta_{0}$. Besides the case $p(t) \equiv p>$ $0, \tau(t)=t-\tau$ or the case $\tau(t)=t-\tau$ and $p(t)$ is $\tau$-periodic, there exists a class of functions which satisfy (10).

Theorem. Consider the differential equation (1) and let $L<1,0<k \leq \frac{1}{e}$ and there exist $\theta>0$ such that

$$
\int_{\tau(u)}^{\tau(t)} p(s) d s \geq \theta \int_{u}^{t} p(s) d s \text { for all } \tau(t) \leq u \leq t .
$$

Assume that

$$
L>\frac{\ln \lambda_{1}+1}{\lambda_{1}}-\frac{1-k-\sqrt{(1-k)^{2}-4 A}}{2},
$$

where $\lambda_{1}$ is the smaller root of the equation $\lambda=e^{k \lambda}$ and $A$ is given by (6). Then all solutions of Eq. (1) oscillate.

Proof. Assume, for the sake of contradiction, that $\mathrm{x}(\mathrm{t})$ is an eventually positive solution of Eq. (1). Then, as in [11, Theorem 1], we obtain

$$
L \leq \frac{\ell n \lambda_{1}+1}{\lambda_{1}}-M
$$

where

$$
M=\liminf _{t \rightarrow \infty} \frac{x(t)}{x(\tau(t))} .
$$

The last inequality, in view of Lemma 2, contradicts (11). The proof is complete.

Remark 2. Observe that when $\theta=1$, then

$$
A=\frac{\lambda_{1}-\lambda_{1} k-1}{\lambda_{1}^{2}}
$$

and (11) reduces to

$$
L>2 k+\frac{2}{\lambda_{1}}-1 .
$$


In the case that $k=\frac{1}{e}$, then $\lambda_{1}=e$ and (12) leads to

$$
L>\frac{4}{e}-1 \approx 0.471517764 .
$$

Example. Consider the delay differential equation

$$
x^{\prime}(t)+p x\left(t-a \sin ^{2} \sqrt{t}-\frac{1}{p e}\right)=0,
$$

where $p>0, a>0$ and $p a=\frac{1}{2}-\frac{1}{e}$. Then

$$
k=\liminf _{t \rightarrow \infty} \int_{\tau(t)}^{t} p d s=\liminf _{t \rightarrow \infty} p\left(a \sin ^{2} \sqrt{t}+\frac{1}{p e}\right)=\frac{1}{e}
$$

and

$$
L=\limsup _{t \rightarrow \infty} \int_{\tau(t)}^{t} p d s=\limsup _{t \rightarrow \infty} p\left(a \sin ^{2} \sqrt{t}+\frac{1}{p e}\right)=p a+\frac{1}{e}=\frac{1}{2} .
$$

Thus, according to Remark 2, all solutions of Eq. (13) oscillate. Observe that none of the results mentioned in the introduction can be applied to this equation.

\section{ACKNOWLEDGEMENT}

The authors would like to thank the referee for some useful comments.

\section{REFERENCES}

[1] O. Arino, G. Ladas and Y. G. Sficas, On oscillations of some retarded differential equations, SIAM J. Math. Anal. 18 (1987), 64-73. MR 88c:34088

[2] J. Chao, On the oscillation of linear differential equations with deviating arguments, Math. in Practice and Theory 1 (1991), 32-40.

[3] Q. Chuanxi and G. Ladas, Oscillations of Neutral Differential Equations with Variable Coefficients, Applicable Anal. 32 (1989), 215-228. MR 90m:34142

[4] Y. Domshlak, Sturmian Comparison Method in investigation of the behavior of solutions for Differential-Operator Equations, "Elm", Baku, USSR, 1986 (Russian).

[5] Y. Domshlak and I. P. Stavroulakis, Oscillations of first-order delay differential equations in a critical state, University of Ioannina, T. R. $N^{0} 257$ November 1995, Applicable Anal. 61 (1996), 359-371. MR 99a:34184

[6] J. Dzurina, Oscillation of second-order differential equations with mixed argument, J. Math. Anal. Appl. 190 (1995), 821-828. MR 95k:34100

[7] Á. Elbert and I. P. Stavroulakis, Oscillations of first order differential equations with deviating arguments, University of Ioannina, T. R. $N^{0} 172$ 1990, Recent trends in differential equations 163-178, World Sci. Ser. Appl. Anal., 1, World Sci. Publishing Co. (1992). MR 93g:34019

[8] Á. Elbert and I. P. Stavroulakis, Oscillation and Non-oscillation Criteria for Delay Differential Equations, University of Ioannina, T. R. $N^{0}$ 228, July 1993, Proc. Amer. Math. Soc. 123 (1995), 1503-1510. MR 95f:34099

[9] L. H. Erbe and B. G. Zhang, Oscillation for first order linear differential equations with deviating arguments, Differential Integral Equations 1 (1988), 305-314. MR 89e:34116

[10] N. Fukagai and T. Kusano, Oscillation theory of first order functional differential equations with deviating arguments, Ann. Mat. Pura Appl. 136 (1984), 95-117. MR 86b:34135

[11] J. Jaroš and I. P. Stavroulakis, Oscillation tests for delay equations, Rocky Mountain J. Math. 29 (1999), 197-207. CMP 99:12

[12] R. G. Koplatadze, On zeros of solutions of first order delay differential equations, Proceedings of I. N. Vekua Institute of Applied Mathematics 14 (1983), 128-135 (Russian). MR 85g:34062

[13] R. G. Koplatadze and T. A. Chanturija, On oscillatory and monotonic solutions of first order differential equations with deviating arguments, Differential'nye Uravnenija 18 (1982), 14631465 (Russian). MR 83k:34069 
[14] R. G. Koplatadze and G. Kvinikadze, On the oscillation of solutions of first order delay differential inequalities and equations, Georgian Math. J. 1 (1994), 675-685. MR 95j:34103

[15] E. Kozakiewicz, Conditions for the absence of positive solutions of a first order differential inequality with a single delay, Archivum Mathematicum, 31 (1995), 291-297. MR 97c:34133

[16] M. K. Kwong, Oscillation of first order delay equations, J. Math. Anal. Appl. 156 (1991), 274-286. MR 92b:34082

[17] G. Ladas, Sharp conditions for oscillations caused by delays, Applicable Anal. 9 (1979), 93-98. MR 80h:34094

[18] G. Ladas, V. Lakshmikantham and L. S. Papadakis, Oscillations of higher-order retarded differential equations generated by the retarded arguments, Delay and Functional Differential Equations and their Applications, Academic Press, New York, 1972 219-231. MR 52:8615

[19] G. Ladas, Y. G. Sficas and I. P. Stavroulakis, Functional differential inequalities and equations with oscillating coefficients, Trends in Theory and Practice of Nonlinear Differential Equations, (Arlington, Tx. 1982), 277-284, Lecture Notes in Pure and Appl. Math., 90 Marcel Dekker, New York, 1984. MR 85f:34128

[20] G. Ladas and I. P. Stavroulakis, On delay differential inequalities of first order, Funkcial. Ekvac. 25 (1982), 105-113. MR 83k:34070

[21] B. Li, Oscillations of delay differential equations with variable coefficients, J. Math. Anal. Appl. 192 (1995), 312-321. MR 96c:34152

[22] B. Li, Oscillation of first order delay differential equations, Proc. Amer. Math. Soc. 124 (1996), 3729-3737. MR 97b:34078

[23] A. D. Myshkis, Linear homogeneous differential equations of first order with deviating arguments, Uspehi Mat. Nauk $5 N^{0} 2$ (36) (1950), 160-162 (Russian).

[24] Ch. G. Philos and Y. G. Sficas, An oscillation criterion for first order linear delay differential equations, Canad. Math. Bull. 41 (1998), 207-213. MR 99b:34119

[25] J. S. Yu and Z. C. Wang, Some further results on oscillation of neutral differential equations, Bull. Austral. Math. Soc. 46 (1992), 149-157. MR 93e:34099

[26] J. S. Yu, Z. C. Wang, B. G. Zhang and X. Z. Qian, Oscillations of differential equations with deviating arguments, PanAmerican Math. J. 2 (1992), 59-78. MR 93e:34100

Department of Mathematics, Boston University, Boston, Massachusetts 02215

E-mail address: mkon@math.bu.edu

Department of Mathematics, University of Ioannina, 45110 Ioannina, Greece

Department of Mathematics, University of Ionnnina, 45110 Ioannina, Greece

E-mail address: ipstav@cc.uoi.gr 